Case Report

\title{
Acute Intermittent Porphyria with Posterior Reversible Encephalopathy Syndrome in Pregnancy: A Case Report
}

\author{
Hemalata Arora ${ }^{1}$, Gaurav Baheti ${ }^{1}$, Ankur Jain ${ }^{1}$, Maulik Bhalsod², Varshil Mehta ${ }^{1}$ \\ ${ }^{1}$ Department of Medicine, Seven Hills Hospital, Mumbai, India, ${ }^{2}$ Department of Radiology, Seven Hills \\ Hospital, Mumbai, India \\ Address for correspondence: Varshil Mehta, Department of Internal Medicine, Seven Hills Hospital, \\ Andheri East, Mumbai 400059, India. E-mail: varshilmehta@gmail.com
}

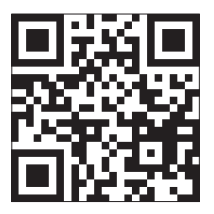

Quick Access Code

How to cite this article: Arora H, Baheti G, Jain A, Bhalsod M, Mehta V. Acute Intermittent Porphyria with Posterior Reversible Encephalopathy Syndrome in Pregnancy: A Case Report. Journal of Medical Research and Innovation.

2019;3(1):e000142.

DOI: 10.15419/jmri.142

Publication history: Received: 24-07-2018 Accepted: 19-09-2018

Published: 19-09-2018

Editors: Dr. Shakti Goel

Copyright: Arora H, This is an open access article distributed under the terms of the Creative Commons Attribution License CC-BY 4.0., which permits unrestricted use, distribution, and reproduction in any medium, provided the original author and source are credited.

Funding: NIL Conflict of Interest: NIL

\section{Abstract}

The porphyrias are a clique of metabolic disorders caused by a faulty heme synthesis process. The acute intermittent porphyria (AIP) is the most common type of porphyria. It results from a decrease in the porphobilinogen deaminase enzyme levels which plays a vital role in the hepatic phase of heme synthesis. A 26-years-old female without any comorbidity or prior history of any disease came with acute abdominal pain. The causes of acute abdominal pain such as the ectopic pregnancy and other causes were excluded after investigations. She was eventually diagnosed with AIP and managed accordingly. The findings of magnetic resonance imaging brain were suggestive of posterior reversible encephalopathy syndrome. The patient was treated with hematin, and glucose and symptomatic treatment were also given. However, the patient had persistent autonomic dysfunction and hyponatremia, followed by cardiac arrest and death. The diagnosis of AIP is often missed in cases of an acute abdominal pain in pregnant women. Early diagnosis of AIP during pregnancy followed by proper management is associated with favorable maternal and fetal outcome. Delay in the management leads to the worse outcomes such as maternal morbidity, mortality, or fetal loss.

Keywords: Acute intermittent porphyria, Pregnancy, Abdominal pain

\section{Introduction}

The porphyrias are a herd of metabolic disorders caused due to the deficiency of various enzymes taking part in the synthesis of heme. Individuals suffering from porphyrias show an accumulation of precursors (porphyrins) of heme synthesis, which are hazardous to skin, liver, nerves, and other organ systems at higher concentrations. The acute intermittent porphyria (AIP) is the most common type of porphyria. In AIP, heme biosynthesis is dysfunctional due to the deficiency of porphobilinogen deaminase (PBG-D), which causes an excessive overproduction and accumulation of porphyrin precursors in the body. ${ }^{[1]}$

The AIP presents with acute attacks which are often provoked by certain drugs (barbiturates and sulfa), starvation, foods, alcohol, chemicals, etc. The hematin is the mainstay of treatment for AIP. It has a negative feedback on the heme synthetic pathway, which prevents synthesis and accumulation of excess heme precursors, and helps in reducing the severity of symptoms during the acute attacks. ${ }^{[2]}$

The abdominal pain is a common presenting symptom in both pregnant females and patients with AIP. The prevalence of AIP $(1 / 20,000$ in Europe) and its incidence are very rare in pregnant females as compared to other causes of abdominal pain (such as ectopic pregnancy, pelvic inflammatory diseases, acute appendicitis, ovarian torsion, and abscess), and hence, AIP during pregnancy is often missed. ${ }^{[3]}$ Furthermore, the line of the management plan for AIP and other causes of abdominal pain during pregnancy are quite different. Even few procedures such 
as laparoscopy may precipitate AIP acute attacks. Many drugs used for pain, autonomic dysfunction, and other symptomatic/ supportive treatment in porphyria are contraindicated in pregnancy. Hence, it is important to diagnose the AIP in pregnancy and treat with caution. ${ }^{[3]}$

\section{Case Report}

A 26-year-old female came to our hospital with complaint of 6 weeks of amenorrhea in association with 1 week of nausea and vomiting and abdominal pain. On examination, the patient had tachycardia (108/min), tachypnea (26/min), and high blood pressure $(160 / 100 \mathrm{mmHg})$. There was no history of fever and urinary complaints. Even the meningeal signs were absent, and plantar was flexor and no neurological deficient noted. The abdomen was soft and non-tender. The ultrasonography of the abdomen and pelvis was suggestive of adnexal mass on the left side with hypertrophied endometrium. The beta-HCG was raised $(1259 \mathrm{mIU} / \mathrm{ml})$. The liver enzymes and C-reactive protein were minimally raised, and serum sodium was low (130 mg/L); however, total leukocyte count, platelets, prothrombin time, and creatinine were within normal limits. The urine sample was negative for proteins and pus cells. In high suspicion of ectopic pregnancy, emergency laparoscopy was done on the day of admission itself; however, intrauterine pregnancy with small ovarian cyst was observed.

The patient developed post-procedure new episodes of seizures. The magnetic resonance imaging (MRI) brain was conducted on the $2^{\text {nd }}$ day and was suggestive of bilateral symmetric T2 and FLAIR hyperintense signal in parietooccipital and bilateral frontal (predominantly watershed) and bilateral cerebellum, leading to a possibility of posterior reversible encephalopathy syndrome (PRES) [Figure 1]. In view

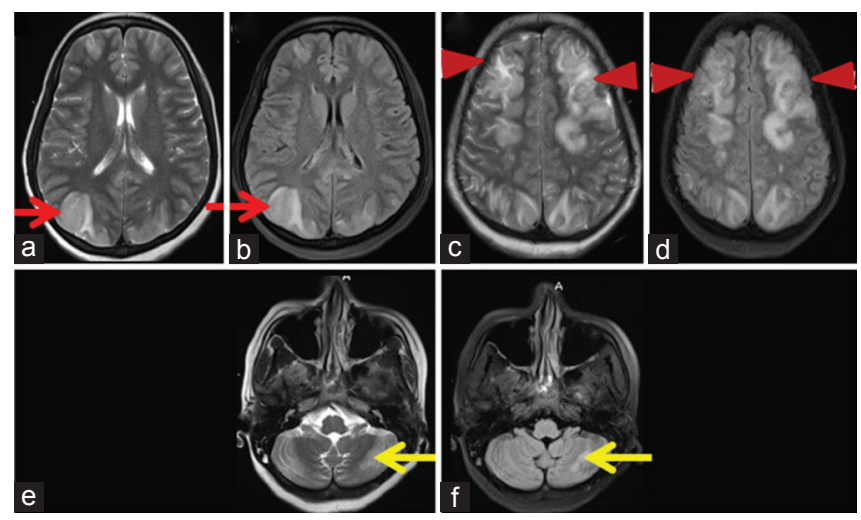

Figure 1: Magnetic resonance imaging brain. Bilateral symmetric T2 (a) and FLAIR (b) hyperintense signal (red arrow) noted in parietooccipital region. Bilateral symmetric T2 (c) and FLAIR (d) hyperintense signal (red arrowhead) noted in the bilateral frontal (predominantly watershed) regions. Subtle T2 (e) and FLAIR (f) hyperintensities (yellow arrow) also noted in the bilateral cerebellum) of abdominal pain with central nervous system involvement, urine sample was sent for urine PBG and aminolevulinic acid (ALA), and the patient was started on high dose glucose (300 g) per day (as an initial treatment plan as we suspected AIP). Meanwhile, patient's tachypnea worsen and she was kept on non-invasive ventilation. She had episodes of night terrors and hallucinations during the course. Urine PBG and ALA levels were high. The patient was started on $4 \mathrm{~g} / \mathrm{kg}$ hematin. The abdominal pain was reduced significantly in next 2 days, but the patient had persistent behavior changes, hyponatremia, and blood pressure fluctuations. She had spontaneous abortion on the $4^{\text {th }}$ day post-admission followed by a cardiac arrest and could not be revived after the event.

\section{Discussion}

The AIP is the most common type of porphyria worldwide with an incidence of 5 in 1,00,000. ${ }^{[4]}$ The prevalence of AIP in the psychiatric population is higher, i.e., 210 cases per lakh population. ${ }^{[5]}$ The most common clinical feature of AIP is a severe abdominal pain, which can be associated with other gastrointestinal manifestations such as vomiting, constipation, or loose stools. The autonomic dysfunction, fever, limb pain, and weakness may develop during the course. The only enzyme deficiency is insufficient to cause an acute attack of AIP. Most of the patients with deficiency of enzymes may never present with any symptoms ("latent" AIP), while some of them may have incidental symptoms. ${ }^{[6]}$ Usually, the acute attacks are triggered by starvation or low carbohydrate diet, drugs, chemicals, stress, etc. These attacks generally often resolve within 2-3 weeks.

The AIP is diagnosed by history, clinical symptoms, and PBG levels in the urine. During the acute phase, the color of urine may turn red or port wine due to porphyrins present in urine which may be the cornerstone in diagnosing it. ${ }^{[6]}$

The treatment of acute attack of AIP consists of symptomatic treatment for pain, nausea, vomit, and hypertension (with drugs considered safe in pregnancy). The disease-specific treatment with hematin should be started if available. The hematin has a negative feedback on the heme synthetic pathway, which prevents synthesis and accumulation of excess heme precursors. Carbohydrate-rich diet and hypertonic infusion of glucose are given if hematin is not available or as initial treatment. ${ }^{[7]}$

The prognosis of AIP in pregnancy is considered poor. The pregnant patients with AIP have a higher incidence of hypertension (16\%), spontaneous abortion (6-12\%), maternal mortality rate (2-4\%), and increased perinatal mortality (2-42\%). ${ }^{[6,8]}$

PRES is characterized by acute neurological symptoms which also includes acute headache, altered mental status, vision loss, and coma. The porphyria is one of the etiological factors 
of PRES. ${ }^{[9]}$ Any features which may suggest that the patient is suffering from PRES should be dealt with utmost care and acutely. The physician should always keep PRES as a differential in his mind in treating any patient with neurological signs and symptoms. The PRES, however, is a misnomer, as cerebral edema can occur in any part of cerebral cortex as was observed in the present case.

\section{Conclusion}

The AIP should be a differential diagnosis in pregnant females with acute abdominal pain and in the absence of any surgical or gynecological causes of abdominal pain. The delay in diagnosis and treatment can increase the mortality and morbidity despite proper management if given late.

While treating the patient symptomatically in AIP with pregnancy with pharmacological agents, one should be more cautious as most of the commonly used drugs can precipitate AIP, and among the safe drugs for AIP, many are contraindicated in pregnancy.

PRES should be ruled out in patient diagnosed with AIP with neurological symptoms such as headache, altered mental status, and coma.

\section{References}

1. Ackner B, Cooper JE, Gray CH, Kelly M. Acute porphyria: A neuropsychiatric and biochemical study. J Psychosom Res 1962;6:1-24.
2. Stein P, Badminton M, Barth J, Rees D, Stewart MF, British and Irish Porphyria Network. et al. Best practice guidelines on clinical management of acute attacks of porphyria and their complications. Ann Clin Biochem 2013;50:217-23.

3. Sunday-Adeoye I, Twomey D, Egwuatu EV, Okonta PI. A 30year review of advanced abdominal pregnancy at the mater misericordiae hospital, Afikpo, Southeastern Nigeria (1976-2006). Arch Gynecol Obstet 2011;283:19-24.

4. Elder G, Harper P, Badminton M, Sandberg S, Deybach JC. The incidence of inherited porphyrias in Europe. J Inherit Metab Dis 2013;36:849-57.

5. Tishler PV, Woodward B, O'Connor J, Holbrook DA, Seidman LJ, Hallett M, et al. High prevalence of intermittent acute porphyria in a psychiatric patient population. Am J Psychiatry 1985;142:1430-6.

6. Martinez N, Nieto A, Guzman M, Heron S, Zapico A. Porphyria and pregnancy. Case report. Colomb Med 2011;42:107-10.

7. Anderson KE, Bloomer JR, Bonkovsky HL, Kushner JP, Pierach CA, Pimstone NR, et al. Recommendations for the diagnosis and treatment of the acute porphyrias. Ann Intern Med 2005;142:439-50.

8. Reshmi VP, Praveen V, Nair VK, Raghavan M. A rare case report of acute intermittent porphyria complicating pregnancy. Indian J Res 2015;4:354-5.

9. Takata T, Kume K, Kokudo Y, Ikeda K, Kamada M, Touge $\mathrm{T}$, et al. Acute intermittent porphyria presenting with posterior reversible encephalopathy syndrome, accompanied by prolonged vasoconstriction. Intern Med 2017;56:713-7. 